\title{
MODELING OF PATIENT FLOWS IN A LARGE-SCALE OUTPATIENT HOSPITAL WARD BY MAKING USE OF ELECTRONIC MEDICAL RECORDS
}

\author{
Soemon Takakuwa
}

\author{
Graduate School of Economics and Business \\ Administration, Nagoya University Hospital \\ Fro-cho, Nagoya University \\ Chikusa-ku, Nagoya, Aichi, 464-8601, JAPAN
}

Daisuke Katagiri

\author{
Logistics System Planning \\ 2-13-4 Shiba, Daifuku Co., Ltd. \\ Minato-ku, Tokyo, 105-0014, JAPAN
}

\begin{abstract}
All departments of an outpatient hospital ward of Nagoya University hospital were simulated to examine patient flows and congestion. The method of gathering the required data on times for all outpatients and their routes is described in the performing simulation, especially by making use of the electronic medical records. An outpatient visits one or more clinical departments and/or one or more test/inspection rooms, the reception area, and the payment department. In this procedure, a series of data of terminal units and of test/inspection terminals was used to obtain the required input data for performing simulation as well as the electronic medical records. It was found that the proposed procedure was quite effective to perform a simulation of a large-scale hospital to examine patient flows by applying an actual case.
\end{abstract}

\section{INTRODUCTION}

The simulation of hospital systems has been conducted to provide hospital administration with tools that will give them the ability to predict the performance under some operational conditions in conjunction with hospital facilities (Austin and Boxerman 1995; Fetter and Thompson 1965). Especially, hospital emergency departments are frequent topic areas for applying simulations. Recent research has reported on such topics as the patient waiting times, reduction of the throughput time, and how to perform simulation experiments.

Several studies have focused on the patients who are processed at various stages through the emergency department. The patient flow and the throughput time were analyzed inside emergency departments (Garcia, Centeno, and DeCario 1995; Mahapatra et al. 2003; McGuire 1994;Samaha, Armel, and Starks 2003). In addition, the issues of scheduling the emergency-department staff were treated for analysis, and were reported in several studies
(Centeno et al. 2003; Draeger 1992; Evans, Gor, and Unger 1996). The issues focusing on simulation models and designs were treated (Miller, Ferrin, and Szymanski 2003; Wiinamaki and Dronzek 2003).

In this present study, a simulation model of the entire departments of the outpatient hospital ward of a university hospital was constructed and used to examine patient flows, especially the patient waiting times. The experimental data to be created included the arrival time of the patient and the patient type, based on the actual data. First, the time intervals spent at each stage for outpatients were measured, where the patients wait for available doctors, and test and inspection, and where they are processed at the medical treatments. Second, the patient waiting time was examined. In this study, a method of gathering required data on times for all outpatients and their routes is described to perform simulation, especially by making use of electronic medical records. Following this, the study shows the special-purpose data-generator designed to create experimental data to execute simulation. Through a series of simulation experiments, the patient waiting time and the congestion inside the hospital can be examined by applying the data generator.

\section{NAGOYA UNIVERSITY HOSPITAL}

The graduate School of Medicine of Nagoya University has a university hospital which comprises outpatient as well as inpatient wards with 29 clinical departments and 30 central clinical facilities. The university hospital has served as a general hospital. The average number of outpatients was 2061.5 and inpatients 844.1 persons in the fiscal year of 2005. The transition of the numbers of outpatients on a day of the week are shown in Figure 1. The mean number of outpatients on Wednesday and Friday are fewer than those on the other days of the week. In addition, the mean number of daily outpatients in the past three years are shown in Figure 2. Nagoya University hospital is planning to rebuild the hospital wards because 
the current building of the hospital remains superannuated. The overall layout of the outpatient hospital ward is shown in Figure 3. The new hospital ward will consist of buildings with three stories above the ground and one below. With the coming new buildings, the number of the patients is certainly expected to increase after completion of the new hospital wards.

\section{A PROCESS OF PATIENTS IN AN OUTPATIENTS HOSPITAL WARD}

The university hospital has prepared a plan for the new hospital wards. Hence, the data on the current hospital wards are used to evaluate the performance on the new hospital wards in this study. The outline of the patient flows and the associated processes in the clinical departments are shown in Figure 4. Basically, the outpatient arrives at the reception, has a test, and consults a doctor. After that, the patient pays expenses, and goes home.

The electronic medical record is basically used for recording the history of medical treatment for the patient; in this study, it is utilized to prepare input data to perform simulation. A layout plan and the precise work shift of the staff are required for input data, and a time study on the test, inspection and treatment activities should be performed as necessary. The overall flow of the data processing proposed in this study is illustrated in Figure 5, and can be itemized mainly as follows:

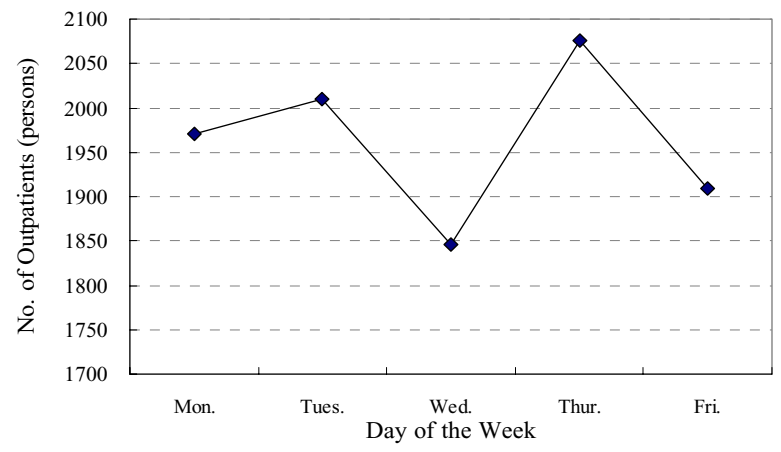

Figure 1: Average Number of Outpatients

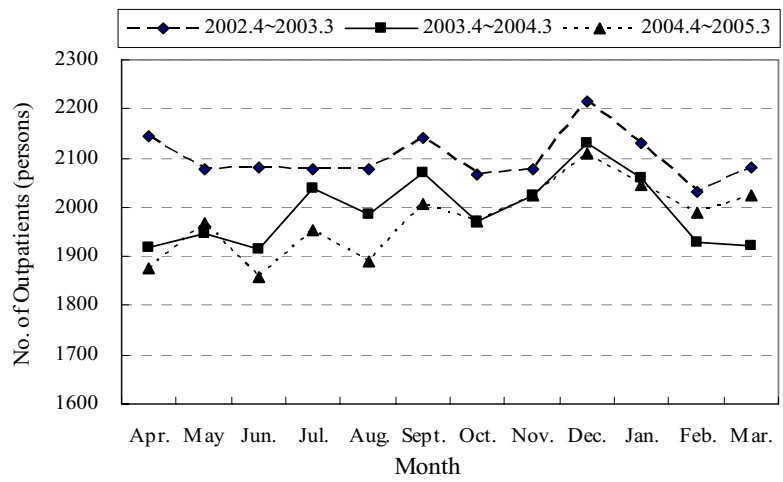

Figure 2: Average Number of Daily Outpatients

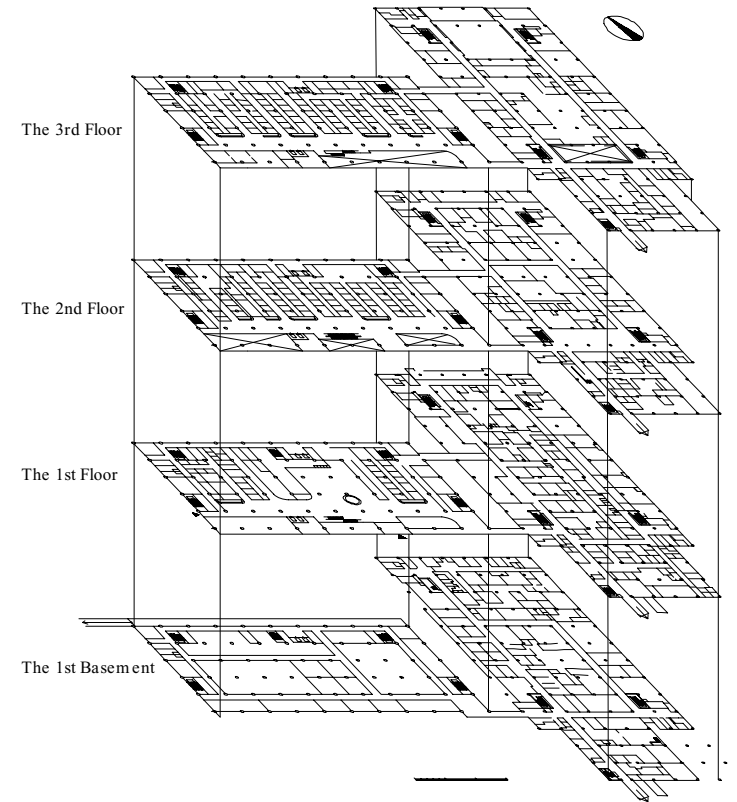

Figure 3: General View of Outpatient Hospital Ward

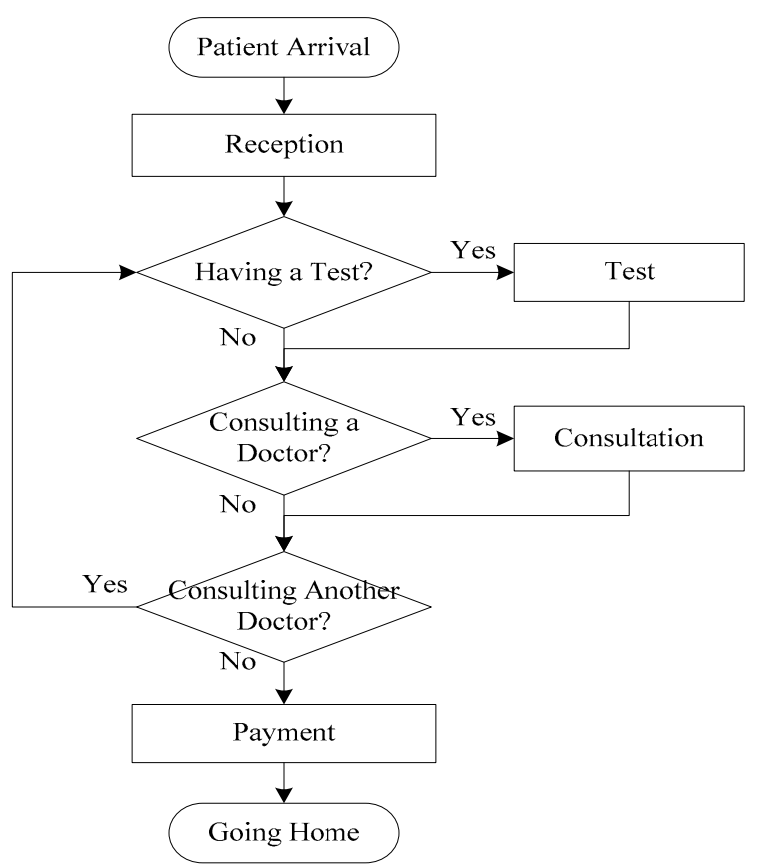

Figure 4: Outline of Outpatient Flows and Processes

(1) Acquire a series of raw data, including reception/payment data, electronic medical record, terminal-unit data, and test/inspection data.

(2) Process the series of raw data.

(3) Prepare input data for simulation, using the data generator.

(4) Perform simulation.

(5) Obtain simulation output. 


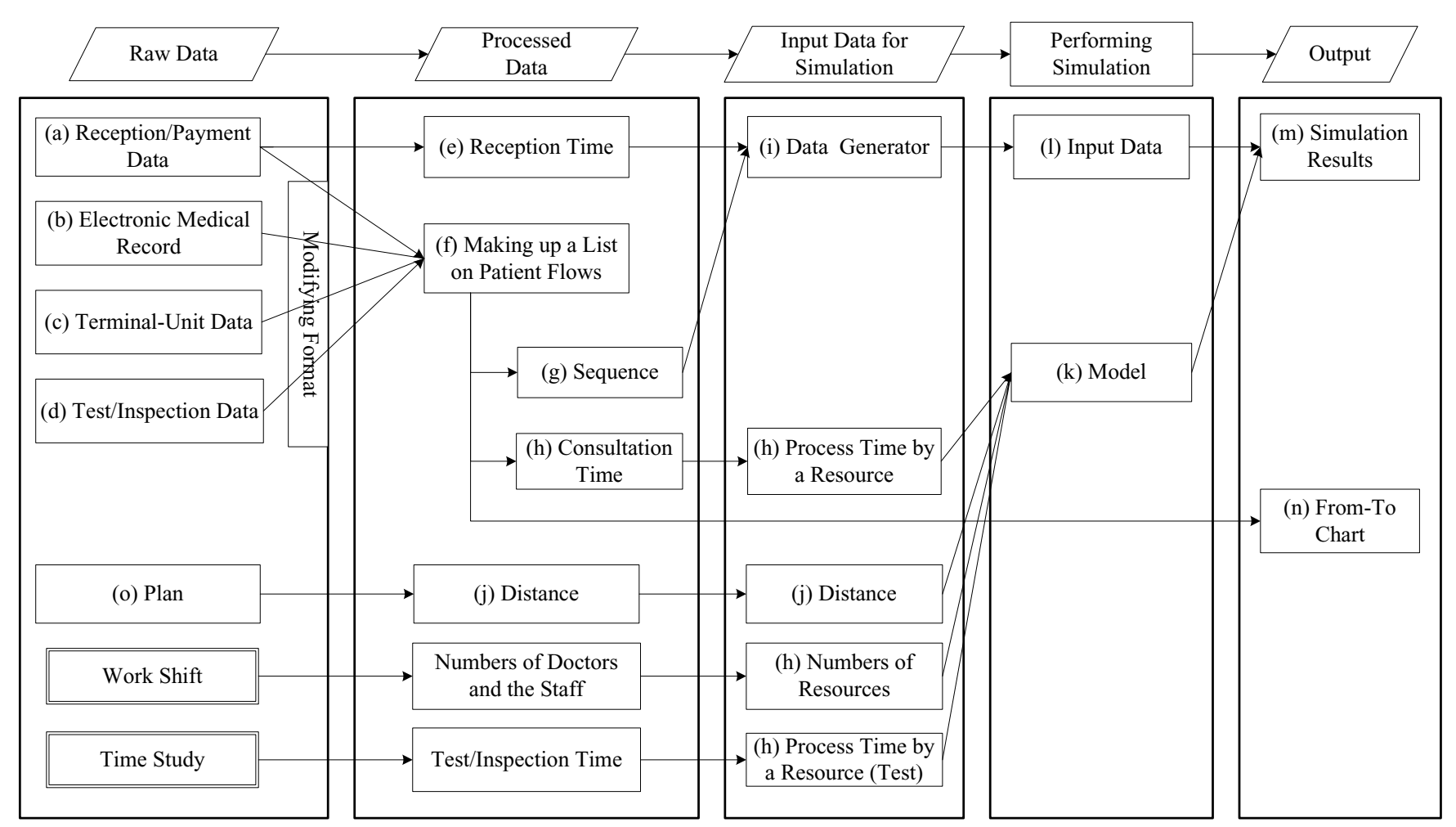

Figure 5: Overall Flow of Data Processing

First, a series of typical sequencing for the routing of approximately eight thousand outpatients was investigated on the electronic medical records for the specified five days. The frequency and the corresponding cumulative percentage for the sequence number are summarized in the ascending order in Figure 6. It is observed that the top 152 sequences account for 72.15 percent of all outpatients. Among them, the most frequent group of sequences are illustrated together with frequency and their routings in Table 1. For example, Sequence Number 90257991 appears 103 times in the electronic medical records of outpatients in five days observation, and its routing comprises the reception, the gastroenterology department, the orthopedic surgery department, and finally payment area.

The clinical departments considered in this study are summarized in Table 2. In this table, the frequency stands for the average number that appeared in the sequences of outpatients in the electronic medical records. In addition, the numbers of the resource unit for clinical departments are summarized. Similarly, the parameters of the receptions of the clinical departments are summarized in Table 3 , and those of the test/inspection departments are summarized in Table 4.

The selected resultant data are shown in Table 5:

(1) Reception/payment data.

(2) Electronic medical record.

(3) Terminal unit.
(4) Test/inspection data.

Blood test/urine test data.

Endoscopy data.

Radiation test.

(5) Number and percentage of accepted patients.

(6) Patient flow.

(7) Sequence.

(8) Resource.

(9) Input data.

In addition, the selected parts of the distances between clinical departments are shown in Table 6. The distances of all possible pairs of routing between the clinical departments and sites should take accurate measurements to perform a simulation.

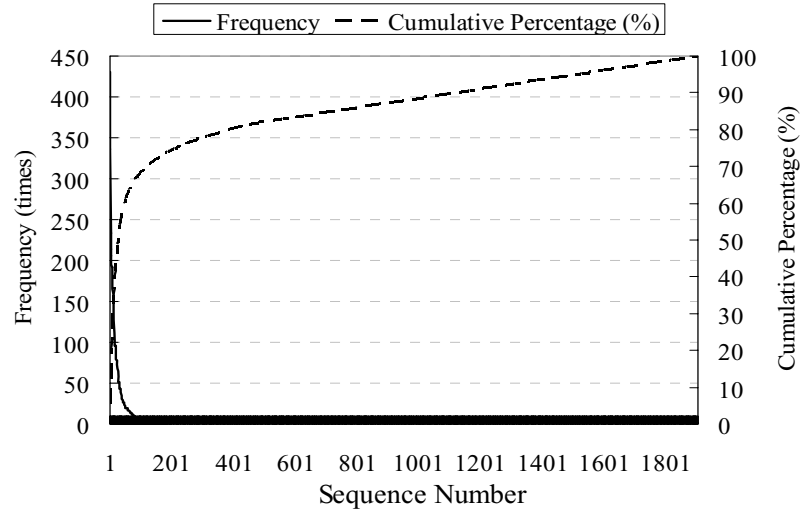

Figure 6: Sequence of Outpatient Flows and Frequency 
Takakuwa and Katagiri

Table 1: The Most Frequent Group of Sequences of Outpatient Flows

\begin{tabular}{|r|r|r|l|l|l|l|}
\hline SeqNo & Frequency & Relative frequency & Destination 1 & Destination 2 & Destination 3 & Destination 4 \\
\hline 904991 & 431 & $7.60 \%$ & Reception & Ophthalmology & Payment & - \\
\hline 905191 & 336 & $5.93 \%$ & Reception & Psychiatry & Payment & - \\
\hline 901091 & 233 & $4.11 \%$ & Reception & Orthopedic Surgery & Payment & - \\
\hline 8291 & 214 & $3.78 \%$ & Radiation Test & Payment & - & - \\
\hline 905491 & 205 & $3.62 \%$ & Reception & Otorhinolaryngology & Payment & - \\
\hline 904791 & 194 & $3.42 \%$ & Reception & Obstetrics and Gynecology & Payment & - \\
\hline 902591 & 191 & $3.37 \%$ & Reception & Gastroenterology & Payment & - \\
\hline 901391 & 188 & $3.32 \%$ & Reception & Urology & Payment & - \\
\hline 902291 & 187 & $3.30 \%$ & Reception & Cardiology & Payment & - \\
\hline 906091 & 165 & $2.91 \%$ & Reception & Dermatology & Payment & - \\
\hline 906491 & 158 & $2.79 \%$ & Reception & Oral and Maxillofacial Surgery & Payment & - \\
\hline 902891 & 138 & $2.44 \%$ & Reception & Neurology & Payment & - \\
\hline 902491 & 130 & $2.29 \%$ & Reception & Diabetology and Endocrinology & Payment & - \\
\hline 902991 & 108 & $1.91 \%$ & Reception & Dept. of General Medicine & Payment & - \\
\hline 7991 & 106 & $1.87 \%$ & Collecting Blood & Payment & - & - \\
\hline 90257991 & 103 & $1.82 \%$ & Reception & Gastroenterology & Orthopedic Surgery & Payment \\
\hline$\ldots$ & $\ldots$ & $\ldots$ & $\ldots$ & $\ldots$ & $\ldots$ \\
\hline$\ldots$ & $\ldots$ & $\ldots$ & $\ldots$ & $\ldots$ & $\ldots$ & \\
\hline
\end{tabular}

Table 2: Parameters on Clinical Departments

\begin{tabular}{|c|c|c|c|c|c|}
\hline Clinical Department & No. & $\begin{array}{c}\text { No. of Units in } \\
\text { Resource }\end{array}$ & \begin{tabular}{|c|} 
Percentage \\
$(\%)$
\end{tabular} & $\begin{array}{c}\text { Consultation Time } \\
\text { (min.) }\end{array}$ & $\begin{array}{c}\text { Frequency } \\
\text { (up to } 5 \text { times }\end{array}$ \\
\hline Orthopedic Surgery & 10 & 7 & \begin{tabular}{|r|}
$4.61 \%$ \\
\end{tabular} & $\operatorname{TRIA}(1,8.8,391)$ & 4.4 \\
\hline Hand Surgery & 11 & 2 & $1.32 \%$ & $\operatorname{TRIA}(1,5.36,97)$ & 22.4 \\
\hline Urology & 13 & 2 & $1.32 \%$ & TRIA(1, 5.68, 179) & 1.6 \\
\hline Radiology & 15 & 3 & $1.97 \%$ & $\operatorname{TRIA}(1,6.06,92)$ & 6.0 \\
\hline Dept. of Emergency Medicine & 17 & 3 & $1.97 \%$ & $\operatorname{TRIA}(1,16.4,370)$ & 8.8 \\
\hline Internal Medicine (Pre-Examination) & 20 & 1 & $0.66 \%$ & TRIA(1, 9.12, 212) & 4.8 \\
\hline Cardiology & 22 & 5 & $3.29 \%$ & $\operatorname{TRIA}(1,6.46,252)$ & 57.4 \\
\hline Nephrology & 23 & 2 & $1.32 \%$ & $\operatorname{TRIA}(1,7.54,171)$ & 18.8 \\
\hline Diabetology and Endocrinology & 24 & 5 & $3.29 \%$ & TRIA(0.999, 5.25, 188) & 54.2 \\
\hline Gastroenterology & 25 & 5 & $3.29 \%$ & $\operatorname{TRIA}(1,5.33,209)$ & 80.6 \\
\hline Hematology & 26 & 3 & $1.97 \%$ & $\operatorname{TRIA}(1,7.73,149)$ & 14.8 \\
\hline Respiroligy & 27 & 3 & $1.97 \%$ & $\operatorname{TRIA}(1,11,322)$ & 17.8 \\
\hline Neurology & 28 & 3 & $1.97 \%$ & $\operatorname{TRIA}(2,6.93,150)$ & 33.6 \\
\hline Dept. of General Medicine & 29 & 3 & $1.97 \%$ & TRIA(1, 9.07, 227) & 25.6 \\
\hline Geriatrics & 30 & 2 & $1.32 \%$ & \begin{tabular}{|l}
- \\
\end{tabular} & \\
\hline Dept. of Outpatient and Home Medicine & 31 & 2 & $1.32 \%$ & TRIA(1, 10.8, 236) & 17.4 \\
\hline Surgery Treatment & 33 & 1 & $0.66 \%$ & $\operatorname{TRIA}(1,4,31)$ & 0.0 \\
\hline Cardiac Surgery & 34 & 2 & $1.32 \%$ & TRIA(1, 5.9, 99) & 5.4 \\
\hline Gastroenterological Surgery & 35 & 4 & $2.63 \%$ & TRIA(1, 5.79, 164) & 30.4 \\
\hline Breast and Endocrine Surgery & 36 & 2 & $1.32 \%$ & $\operatorname{TRIA}(1,7,109)$ & 4.2 \\
\hline Pediatric Surgery & 37 & 1 & $0.66 \%$ & $\operatorname{TRIA}(1,7.18,137)$ & 10.6 \\
\hline Vascular Surgery & 38 & 2 & $1.32 \%$ & $\operatorname{TRIA}(1,15.5,233)$ & 6.2 \\
\hline Thoracic Surgery & 39 & 1 & $0.66 \%$ & $\operatorname{TRIA}(3,14.2,115)$ & 0.0 \\
\hline Transplantation Surgery & 40 & 1 & $0.66 \%$ & $\operatorname{TRIA}(1,15.6,147)$ & 0.0 \\
\hline Neurosurgery & 42 & 3 & $1.97 \%$ & $\operatorname{TRIA}(1,5.41,142)$ & 27.0 \\
\hline Obstetrics and Gynecology & 47 & 4 & $2.63 \%$ & TRIA( $(1,10.3,373)$ & 64.6 \\
\hline Ophthalmology & 49 & 5 & $3.29 \%$ & TRIA(1, 5.21, 203) & 94.8 \\
\hline Psychiatry & 51 & 3 & $1.97 \%$ & $\operatorname{TRIA}(1,7.45,272)$ & 75.8 \\
\hline Dept. od Psychiatry for Parents and Children & 52 & 2 & $1.32 \%$ & TRIA(1, 9.55, 189) & 19.4 \\
\hline Otorhinolaryngology & 54 & 6 & $3.95 \%$ & $\operatorname{TRIA}(1,6.03,202)$ & 51.4 \\
\hline Anesthesiology & 56 & 1 & $0.66 \%$ & TRIA(0.999, 9.9, 179) & 16.0 \\
\hline Pediatrics & 58 & 5 & $3.29 \%$ & $\operatorname{TRIA}(1,6.44,175)$ & 32.4 \\
\hline Dermatology & 60 & 4 & $2.63 \%$ & TRIA(1, 6.67, 228) & 44.8 \\
\hline Plastic and Reconstructive Surgery & 62 & 2 & $1.32 \%$ & TRIA(0.999, 13.7, 204) & 8.8 \\
\hline Oral and Maxillofacial Surgery & 64 & 6 & $3.95 \%$ & $\operatorname{TRIA}(1,9.81,318)$ & 43.2 \\
\hline Dept. of Surgical Center & 77 & 1 & $0.66 \%$ & $\operatorname{TRIA}(1,8.9,80)$ & 0.0 \\
\hline Dept. of Physiatrics & 85 & 3 & $1.97 \%$ & $\operatorname{TRIA}(1,14.5,136)$ & 0.0 \\
\hline
\end{tabular}




\section{Takakuwa and Katagiri}

Table 3: Parameters on Reception/Machines

\begin{tabular}{|l|r|r|r|l|r|}
\hline \multirow{2}{*}{ Reception/Machine } & \multirow{2}{*}{ No. } & No. of Units in & Percentage & Processing Tome & Frequency \\
\cline { 3 - 6 } & & Resource & $(\%)$ & $($ min. $)$ & (up to 5 times \\
\hline Orthopedic Surgery & 10 & 1 & $0.66 \%$ & TRIA $(0.999,3.28,33)$ & 95.2 \\
\hline Urology & 12 & 1 & $0.66 \%$ & TRIA $(1,4.1,32)$ & 52.4 \\
\hline Radiology & 14 & - & $0.00 \%$ & - & -1.2 \\
\hline Dept. of Emergency Medicine & 16 & - & $0.00 \%$ & TRIA $(1,3.33,29)$ & 1 \\
\hline Internal Medicine & 19 & 1 & $0.66 \%$ & TRIA $(1,2.45,33)$ & 4.6 \\
\hline Surgery & 32 & 1 & $0.66 \%$ & TRIA $(1,2.62,43)$ & 3 \\
\hline Neurosurgery & 41 & 1 & $0.66 \%$ & TRIA $(1,2.83,34)$ & 1.6 \\
\hline Obstetrics and Gynecology & 46 & 1 & $0.66 \%$ & TRIA $(1,2.5,16)$ & 8.6 \\
\hline Ophthalmology & 48 & - & $0.66 \%$ & TRIA $(1,2.86,27)$ & 8.2 \\
\hline Psychiatry & 50 & 1 & $0.66 \%$ & TRIA $(1,3.79,40)$ & 10.2 \\
\hline Otorhinolaryngology & 53 & 1 & $0.66 \%$ & TRIA $(1,3.29,33)$ & - \\
\hline Anesthesiology & 55 & - & $0.00 \%$ & - & 13.4 \\
\hline Pediatrics & 57 & 1 & $0.66 \%$ & TRIA $(1,5.23,94)$ & 8.2 \\
\hline Dermatology & 59 & 1 & $0.66 \%$ & TRIA $(1,2.67,31)$ & - \\
\hline Plastic and Reconstructive Surgery & 61 & 1 & $0.66 \%$ & - & - \\
\hline Oral and Maxillofacial Surgery & 63 & 1 & $0.66 \%$ & TRIA $(1,2.6,17)$ & 1128.6 \\
\hline Payment Machine & 90 & 5 & $3.29 \%$ & TRIA $(19,32.9,80)$ & 1005.2 \\
\hline Reexamining Reception Machine & 91 & 5 & $3.29 \%$ & TRIA $(0.5,1,1.5)$ & \\
\hline
\end{tabular}

Table 4: Parameters on Clinical and Test/Inspection Departments

\begin{tabular}{|l|r|r|r|c|r|}
\hline \multirow{2}{*}{ Clinical Dept. } & \multirow{2}{*}{ No. } & No. Units in & Percentage & Treatment Time & \multicolumn{1}{c|}{ Frequency } \\
\cline { 3 - 6 } & & Resource & $(\%)$ & $(\mathrm{min})$. & (up to 5 times \\
\hline Collecting Blood & 79 & 5 & $3.29 \%$ & TRIA $(0.07,1.8,7)$ & 207.6 \\
\hline Urine Test & 80 & 1 & $0.66 \%$ & $\operatorname{TRIA}(0.5,1,1.5)$ & 35.8 \\
\hline Endoscopy & 81 & 1 & $0.66 \%$ & TRIA $(2.03,2.93,11)$ & 36.2 \\
\hline Radiation Test & 82 & 5 & $3.29 \%$ & TRIA $(1,1.66,8.9)$ & 115.8 \\
\hline
\end{tabular}

In case more patients are expected to be processed at the clinical departments, it is necessary to perform simulation experiments under any possible situation. Hence, a special-purpose data-generator was designed and developed to create experimental data in order to examine more congested situations, taking the current situation in the emergency department. This data generator was written in Excel VBA. Experimental data created consisting of the arrival time of the patient, the arrival time, and the route sequences (Takakuwa and Shiozaki 2004; Wijewickrama and Takakuwa 2005; Wijewickrama and Takakuwa 2006). A similar idea for the data generator for simulation experiments appears in simulation of warehousing at distribution centers and at the internationaldeparture airport (Takakuwa et al. 2000; Takakuwa and Oyama 2003). The generated data includes the arrival time of each patient and the sequence of the routing. By making use of these generated data as an external file input for the simulation model, experiments can be conducted under any specified conditions.

\section{SIMULATION EXPERIMENTS}

In this section, the above-mentioned procedure of preparing simulation data explains the process of obtaining the patient waiting times and consultation times, using numerical examples; consider the case of two thousand patients or the typical congestion in a hospital. In this case, all associated areas of the outpatient hospital ward were included in a simulation model that was used to examine patient flows, and to collect important statistics including all waiting time. The simulation models in this study were created using Arena (Kelton, Sadowski, and Sadowski 2006).

In order to investigate the waiting times, the consultation times, and the degree of congestion inside the hospital ward, the ten replications of the simulation were executed. The $95 \%$ confidence interval on the average percentage of the waiting time and the consultation time for 26 clinical departments are shown in Figures 7 and 8, respectively. The numbers of the clinical departments in these figures correspond to those in Table 2. As expected, 


\section{Takakuwa and Katagiri}

these figures show that the waiting time at the clinical department generally is much longer than the consultation time. The waiting times for consultation of the urology and the psychiatry departments are especially longer compared to those of all clinical departments.

The proposed procedure of gathering required data from electronic medical records is found to be quite effec- tive to perform simulation of a large-scale hospital to examine patient flows and congestion. Furthermore, any situation can be simulated flexibly under the specific scenario by varying the number of expected outpatients and their mix, and/or the number of any resource units at the clinical departments in the hospital.

Table 5: Selected Resultant Data Obtained by Data Processing

(a) Reception/Payment Data

$>$ 10:00:22 Data aquired.(006989772,40, Reception done.,09:58:58)

$>$ 13:54:04 Data aquired.(006989772,40, Payment done.,13:52:42)

\begin{tabular}{|r|l|r|r|}
\hline Patient ID & Clinical Department & Log on & Log off \\
\hline 6989772 & Reception & $9: 58: 58$ & \\
\hline 6989772 & Payment & $13: 52: 42$ & \\
\hline
\end{tabular}

(b) Electronic Medical Record

\begin{tabular}{|c|c|c|c|c|c|c|}
\hline The Date and Time & millisecond & Terminal Unit & - & - & Patient ID & User ID \\
\hline Patient ID & Terminal Unit & Log on & Log off & & & \\
\hline
\end{tabular}

(c) TerminalUnit

\begin{tabular}{|c|c|c|}
\hline Computer Name & TermName & \multicolumn{1}{|c}{} \\
\hline FMV15079 & Outpatient Ward 3rd Fl. & \\
\hline Terminal Unit Name & Consultation Room & Clinical Dept. \\
\hline FMV15079 & Outpatient Ward 3rd Fl. & Ophthalmology \\
\hline
\end{tabular}

(d) Test/Inspection Data

Blood Test/Urine Test Data

\begin{tabular}{|c|c|c|c|c|c|c|}
\hline Department C & Department Name & Patient ID & Order No. & System Date & System Time & User C \\
\hline 40 & Ophthalmology & 00006989772 & 04216938 & 20050801 & 1355 & 01 \\
\hline 40 & Ophthalmology & 00006989772 & 04216938 & 20050801 & 1407 & 02 \\
\hline Patient ID & Clinical Department & Log on & Log off & & & \\
\hline 6989772 & Blood Test & 13:55:00 & & & & \\
\hline 6989772 & Urine Test & 14:07:00 & & & & \\
\hline \multicolumn{7}{|l|}{ doscopy Data } \\
\hline Date & Patient ID & Time & Requested by & Room & & \\
\hline 2005.08.01(Mon.) & 6989772 & $13: 09$ & Ophthalmology & Electrocardiogram Room & & \\
\hline Patient ID & Clinical Department & Log on & Log off & & & \\
\hline 6989772 & Endoscopy & 13:09 & & & & \\
\hline \multicolumn{7}{|c|}{ Radiation Test } \\
\hline Requested by & Date & Patient ID & Time & Clinical Department (Patient) & Room & Appointment \\
\hline Orthopedic Surgery & 2005.08.01 (mon.) & 3216217 & 0:00 & Orthopedic Surgery & No.22 Room & 2005.08.01(Mon. \\
\hline
\end{tabular}

\begin{tabular}{|c|c|c|c|}
\hline Patient ID & Clinical Department & Log on & Log off \\
\hline 6989772 & Radiology & & \\
\hline
\end{tabular}

Time Interval of Reception

\begin{tabular}{|l|r|r|}
\hline \multicolumn{1}{|c|}{ Time Interval } & \multicolumn{1}{|c|}{ Number of patients } & \multicolumn{1}{c|}{ Percentage } \\
\hline $7: 30-8: 00$ & 0 & $0.00 \%$ \\
\hline $8: 00-8: 30$ & 269 & $3.49 \%$ \\
\hline $8: 30-9: 00$ & 669 & $8.68 \%$ \\
\hline $9: 00-9: 30$ & 1088 & $14.11 \%$ \\
\hline $9: 30-10: 00$ & 1031 & $13.37 \%$ \\
\hline $10: 00-10: 30$ & 1013 & $13.14 \%$ \\
\hline $10: 30-11: 00$ & 878 & $11.39 \%$ \\
\hline $11: 00-11: 30$ & 774 & $10.04 \%$ \\
\hline $11: 30-12: 00$ & 511 & $6.63 \%$ \\
\hline $12: 00-12: 30$ & 380 & $4.93 \%$ \\
\hline $12: 30-13: 00$ & 237 & $3.07 \%$ \\
\hline
\end{tabular}

(f) Patient Flow

\begin{tabular}{|c|l|l|l|l|l|l|l|}
\hline Patient ID & \multicolumn{1}{|c|}{ Code 1 } & Clinical Dept. & \multicolumn{1}{c|}{ Code 2 } & \multicolumn{1}{c|}{ Room Name } & Terminal Unit & \multicolumn{1}{|c|}{ Log on } & Log off \\
\hline 0003239 & 86 & Reception & 750 & Reception & 8:45:39 & \\
\hline 0003239 & 38 & Vascular Surgery & 140 & Outpatient Ward 2nd Fl. Surgery No.9 B & FMV05034 & $9: 19: 51$ \\
\hline 0003239 & 41 & Neurosurgery & 147 & Outpatient Ward 2nd Fl. Neurosurgery No.2 & FMV06033 & $9: 23: 51$ \\
\hline 0003239 & 87 & Payment & 751 & Payment & & $10: 42$ \\
\hline
\end{tabular}

(g) Sequence \begin{tabular}{l|l|l|}
\hline Neurosurgery & 147 & Outpatient \\
\hline Payment & 751 & Payment \\
\hline
\end{tabular}

\begin{tabular}{|l|r|r|}
\hline Time Interval & Number of patients & Percentage \\
\hline $13: 00-13: 30$ & 232 & $3.01 \%$ \\
\hline $13: 30-14: 00$ & 183 & $2.37 \%$ \\
\hline $14: 00-14: 30$ & 163 & $2.11 \%$ \\
\hline $14: 30-15: 00$ & 100 & $1.30 \%$ \\
\hline $15: 00-15: 30$ & 82 & $1.06 \%$ \\
\hline $15: 30-16: 00$ & 38 & $0.49 \%$ \\
\hline $16: 00-16: 30$ & 38 & $0.49 \%$ \\
\hline $16: 30-17: 30$ & 18 & $0.23 \%$ \\
\hline $17: 00-17: 30$ & 5 & $0.06 \%$ \\
\hline $17: 30-18: 00$ & 0 & $0.00 \%$ \\
\hline Total & 7709 & $100.00 \%$ \\
\hline
\end{tabular}

\begin{tabular}{|l|r|r|r|r|r|r|}
\hline SeqNo & Frequency & Percentage & Accumulative \% & Destination (1st) & Ditto (2nd) & Ditto (3rd) \\
\hline 904991 & 431 & $7.604 \%$ & $7.604 \%$ & Reception & Ophthalmology & Payment \\
\hline 905191 & 336 & $5.928 \%$ & $13.532 \%$ & Reception & Psychiatry & Payment \\
\hline 901091 & 233 & $4.111 \%$ & $17.643 \%$ & Reception & Orthopedic S. \\
\hline
\end{tabular}

(h) Resource

\begin{tabular}{|l|r|r|l|}
\hline \multicolumn{1}{|c|}{ Clinical Dept. } & No. of Resource Units & Mean Time & Consultation Time \\
\hline Orthopedic S. Reception & 1 & 3.4 & TRIA $(1,3.28,33)$ \\
\hline Orthopedic S. & 7 & 16.9 & TRIA $(1,8.8,391)$ \\
\hline Hand Surgery & 2 & 13.4 & TRIA $(1,5.36,97)$ \\
\hline
\end{tabular}

(i) Hand Surgery

(l) InputData

\begin{tabular}{|l|l|l|l|}
\hline \multicolumn{1}{|c|}{ Patient ID } & \multicolumn{1}{|c|}{ Arrival Time } & & Sequence \\
\hline 140 & 470.3320007 & 1 & 904991 \\
\hline 118 & 472.821991 & 1 & 905191 \\
\hline
\end{tabular}




\section{Takakuwa and Katagiri}

Table 6: Distances between Selected Clinical Departments

\begin{tabular}{|c|c|c|c|c|c|c|c|c|c|c|c|c|c|c|}
\hline & Entrance & R. R. & O. R & $\mathrm{O}$. & P. R. & Payment & N.R. & N. & O. S.R. & O. S. & H. S. & 1F.E. LB & $1 \mathrm{~F} . \mathrm{LB}$ & Exit \\
\hline Entrance & & 20 & 61 & \begin{tabular}{|l|}
71 \\
\end{tabular} & 36 & $\begin{array}{r}36 \\
\end{array}$ & 38 & 58 & \begin{tabular}{|r|}
50 \\
\end{tabular} & 66 & 74 & 58 & \begin{tabular}{|r|}
17 \\
\end{tabular} & 6 \\
\hline Reexamination R. (*) & 20 & & 48 & 59 & 32 & 32 & 33 & 51 & 41 & 60 & 69 & 58 & 18 & 23 \\
\hline Ophthalmology R. & 61 & 48 & 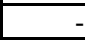 & 27 & 63 & 67 & 67 & 88 & 78 & 95 & 104 & 99 & 58 & 68 \\
\hline Ophthalmology & 71 & 59 & 27 & - & 76 & 80 & 80 & 98 & 93 & 108 & 117 & 115 & 72 & 82 \\
\hline Payment R. & 36 & 32 & 63 & 76 & - & 6 & 26 & 40 & 36 & 53 & 62 & 69 & 36 & 33 \\
\hline Payment & 36 & 32 & 67 & 80 & 6 & - & 23 & 39 & 33 & 51 & 60 & 62 & 41 & 38 \\
\hline Neurosurgery R. & 38 & 33 & 67 & 80 & 26 & 23 & - & 24 & 15 & 32 & 41 & 44 & 36 & 32 \\
\hline Neurosurgery & 58 & 51 & 88 & $\overline{98}$ & 40 & 39 & 24 & - & 26 & 44 & 50 & 57 & 56 & 54 \\
\hline Orthopedic Surgery R. & 50 & 41 & 78 & 93 & 36 & 33 & 15 & 26 & - & 21 & 30 & 35 & 49 & 43 \\
\hline Orthopedic Surgery & 66 & 60 & 95 & 108 & 53 & 51 & 32 & 44 & 21 & - & 20 & 40 & 63 & 58 \\
\hline Hand Surgery & 74 & 69 & 104 & 117 & 62 & 60 & 41 & 50 & 30 & 20 & - & 52 & 74 & 71 \\
\hline 1st Fl. East LB & 58 & 58 & 99 & 115 & 69 & 62 & 44 & 57 & 35 & 40 & 52 & - & 56 & 50 \\
\hline 1 st Fl. LB & 17 & 18 & 58 & 72 & 36 & 41 & 36 & 56 & 49 & 63 & 74 & 56 & - & 23 \\
\hline Exit & 6 & 23 & 68 & 82 & 33 & 38 & 32 & 54 & 43 & 58 & 71 & 50 & 23 & \\
\hline
\end{tabular}

(*)R.: Reception

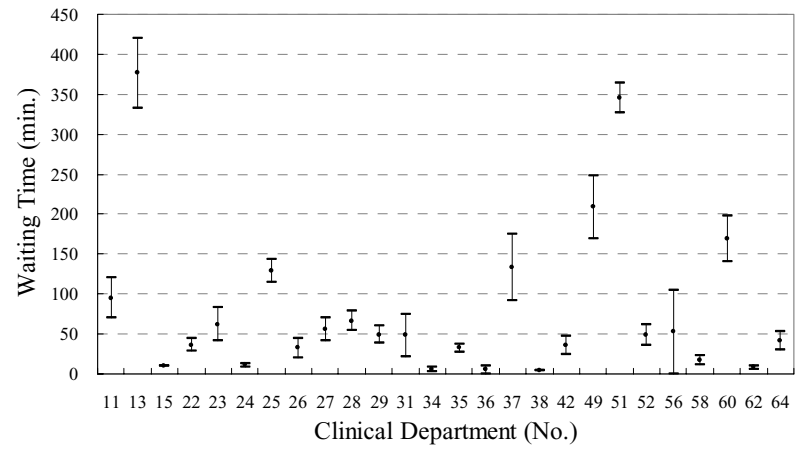

Figure 7: Simulation Results on Waiting Time

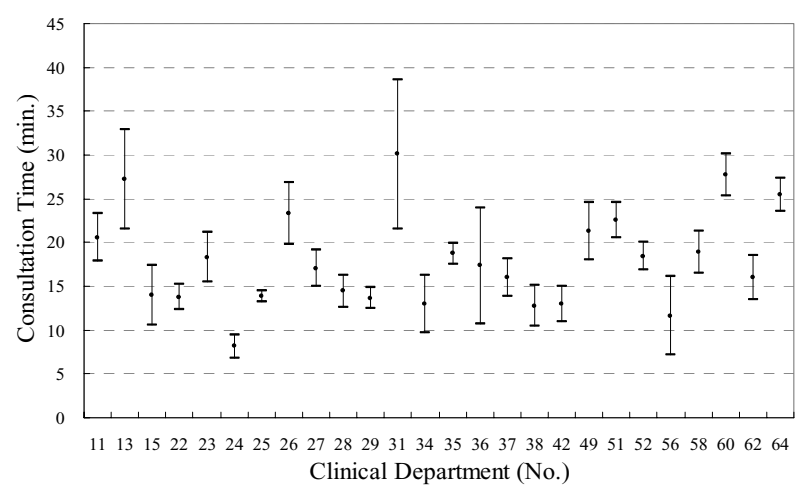

Figure 8: Simulation Results on Consultation Time

\section{SUMMARY}

(1) A simulation model of the planned outpatient ward of a university hospital was constructed and used especially to examine the patient waiting time and congestion.

(2) The method of gathering required data on times for all outpatients and their routes was proposed to perform a simulation, especially by making use of electronic medical records.

(3) The proposed procedure was presented using an actual case to demonstrate the applicability to a large-scale university hospital.

\section{ACKNOWLEDGMENTS}

The authors wish to express sincere gratitude to Dr. Y. Oiso, Deputy Director of Nagoya University Hospital for the acknowledgment of this study.

\section{REFERENCES}

Austin, C. J. and S. B. Boxerman. 1995. Quantitative Analysis for Health Services Administration, Ann Arbor, MI. AUPHA Press/ Health Administration Press.

Centeno, M., R. Giachetti, R. Linn, and A. M. Ismail. 2003. A simulation-ILP based tool for scheduling ER staff. In Proceedings of the 2003 Winter Simulation Conference, ed. S. Chick, P. J. Sanchez, D. Ferrin, and D. J. Morrice, 1930-1937. Piscataway, New Jersey: Institute of Electrical and Electronics Engineers. Available online via <http://www.informssim.org/wsc03papers/251.pdf> [accessed January 31, 2007].

Draeger, M. A. 1992. An emergency department simulation model used to evaluate alternative nurse staffing and patient population scenarios. In Proceedings of the 1992 Winter Simulation Conference, ed. J. J. Swain, D. Goldsman, R. C. Crain, and J. R. Wilson. 1057-1064. Piscataway, New Jersey: Institute of Electrical and Electronics Engineers.

Evans, G. W., T. B. Gor, and E. Unger. 1996. A simulation model for evaluating personnel schedules in a hospital emergency department. In Proceedings of the 1996 Winter Simulation Conference, ed. J. M. 
Charles, D. J. Morrice, D. T. Brunner, and J. J. Swain. 1205-1209. Piscataway, New Jersey: Institute of Electrical and Electronics Engineers.

Fetter, R. B. and J. D. Thompson. 1965. The simulation of hospital systems. Operations Research. SeptemberOctober 1965; 689-711.

Garcia, M. L., M. A. Centeno, C. Rivera, and N. DeCario. 1995. Reducing time in an emergency room via fasttrack. In Proceedings of the 1995 Winter Simulation Conference, ed. C. Alexopoulos, K., Kang, W. R. Lilegdon, and D. Goldsman. 1077-1080. Piscataway, New Jersey: Institute of Electrical and Electronics Engineers.

Kelton,W,D., R.P.Sadowski, and D.A.Sadowski. 2006. Simulation with Arena. 4th ed. New York, NY: McGraw-Hill.

Mahapatra, S., C. P. Koelling, L. Patvivatsiri,, B. Fraticelli, D. Eitel, and L. Grove. 2003. Pairing emergency severity index5-level triage data with computer aided system design to improve emergency department access and throughput. In Proceedings of the 2003 Winter Simulation Conference, ed. S. Chick, P. J. Sanchez, D. Ferrin, and D. J. Morrice, 1917-1925. Piscataway, New Jersey: Institute of Electrical and Electronics Engineers. Available online via <http://www.informssim.org/wsc03papers/249.pdf> [accessed January 31, 2007].

McGuire, F. 1994. Using simulation to reduce length of stay in emergency departments. In Proceedings of the 1994 Winter Simulation Conference, ed. J. D. Tew, S. Manivannan, D. A. Sadowski, and A. F. Seila. 861-867. Piscataway, New Jersey: Institute of Electrical and Electronics Engineers.

Miller, M. J., D. M. Ferrin, and J. M. Szymanski. 2003. Simulating six sigma improvement ideas for a hospital emergency department. In Proceedings of the 2003 Winter Simulation Conference, ed. S. Chick, P. J. Sanchez, D. Ferrin, and D. J. Morrice, 1926-1929. Piscataway, New Jersey: Institute of Electrical and Electronics Engineers. Available online via $<$ http: / / www. informs -

sim.org/wsc03papers/250.pdf> [accessed January 31, 2007].

Samaha, S., W. S. Armel, and D. W. Starks. 2003. The use of simulation to reduce the length of stay in an emergency department. In Proceedings of the 2003 Winter Simulation Conference, ed. S. Chick, P. J. Sanchez, D. Ferrin, and D. J. Morrice, 1907-1911. Piscataway, New Jersey: Institute of Electrical and Electronics Engineers. Available online via <http: / / www. informs- sim.org/wsc03papers/247.pdf> [accessed January 31, 2007].

Takakuwa. S. and T. Oyama. 2003. Simulation analysis of international-departure passenger flows in an airport terminal. In Proceedings of the 2003 Winter Simulation Conference, ed. S. Chick, P. J. Sanchez, D. Ferrin, and D. J. Morrice, 1627-1634. Piscataway, New Jersey: Institute of Electrical and Electronics Engineers. Available online via <http: / / www. informs sim.org/wsc03papers/207.pdf> [accessed January 31, 2007].

Takakuwa, S. and H. Shiozaki. 2004. Functional analysis for operating emergency department of a general hospital. In Proceedings of the 2004 Winter Simulation Conference, ed. R. G. Ingals, M. D. Rossetti, J. S. Smith, and B. A. Peters, 2003-2011. Piscataway, New Jersey: Institute of Electrical and Electronics Engineers. Available online via <http: / / www. informssim.org/wsc04papers / 268.pdf> [accessed January 31, 2007].

Takakuwa, S., H. Takizawa, K. Ito, and S. Hiraoka. 2000. Simulation and analysis of non-automated distribution warehouse. In Proceedings of the 2000 Winter Simulation Conference, ed. J. A. Joines, R. R. Barton, K. Kang, and P. A. Fishwick, 1177-1184. Piscataway, New Jersey: Institute of Electrical and Electronics Engineers. Available online via <http: / / www. informs sim.org/wsc00papers/159.PDF> [accessed January 31, 2007].

Wiinamaki, A. and R. Dronzek. 2003. Using simulation in the architectural concept phase of an emergency department design. In Proceedings of the 2003 Winter Simulation Conference, ed. S. Chick, P. J. Sanchez, D. Ferrin, and D. J. Morrice, 1912-1916. Piscataway, New Jersey: Institute of Electrical and Electronics Engineers. Available online via <http: / / www. informs-

sim.org/wsc03papers/248.pdf> [accessed January 31, 2007].

Wijewickrama, A. and S. Takakuwa. 2005. Simulation analysis of appointment scheduling in an outpatient department of internal medicine. In Proceedings of the 2005 Winter Simulation Conference, ed. M. E. Kuhl, N. M. Steiger, F. B. Armstrong, and J. A. Joines, 2264-2273. Piscataway, New Jersey: Institute of Electrical and Electronics Engineers. Available online via <http://www.informssim.org/wsc05papers/284.pdf> [accessed January 31, 2007].

Wijewickrama, A. and S. Takakuwa. 2006. Simulation analysis of an outpatient department of internal medi- 
cine in a university hospital. In Proceedings of the 2006 Winter Simulation Conference, ed. L. F. Perrone, F. P. Wieland, J. Liu, B. G. Lawson, and R. M. Fujimoto, 425-432. Piscataway, New Jersey: Institute of Electrical and Electronics Engineers. Available online via <http://www.informssim.org/wsc06papers/050.pdf> [accessed January 31,2007$]$.

\section{AUTHOR BIOGRAPHIES}

SOEMON TAKAKUWA is a Professor in the Graduate School of Economics and Business Administration at Nagoya University in Japan. He received his B. Sc. and M. Sc. degrees in industrial engineering from Nagoya Institute of Technology in 1975 and from Tokyo Institute of Technology in 1977 respectively. His Ph.D. is in industrial engineering from The Pennsylvania State University. He holds a Doctorate of Economics from Nagoya University. His research interests include optimization of manufacturing and logistics systems, management information system and system simulation. He has prepared the Japanese editions of both Introduction to simulation using SIMAN and Simulation with ARENA. He has been serving concurrently as the senior staff of Department of Hospital Management Strategy and Planning at Nagoya University Hospital. His e-mail address is <takakuwa@soec.nagoya-u.ac.jp>.

DAISUKE KATAGIRI received his B. Sc. and M. Sc. Degrees in Economics and Business Administration at Nagoya University in 2004 and 2006, respectively. He has been working for Daifuku Co., Ltd. His research interests include the simulation of large-scale general hospitals and logistics systems. His e-mail address is <katagiri.degmail.com>. 